\title{
Cerebral Perfusion Pressure Insults and Associations with Outcome in Adult Traumatic Brain Injury
}

\author{
Fabian Güiza, Geert Meyfroidt, Ian Piper, Giuseppe Citerio, ${ }^{3}$ Iain Chambers, ${ }^{4}$ Per Enblad, \\ Pelle Nillson, Bart Feyen, ${ }^{6}$ Philippe Jorens, Andrew Maas, Martin U. Schuhmann, \\ Rob Donald, Laura Moss, ${ }^{10}$ Greet Van den Berghe, and Bart Depreitere ${ }^{11}$
}

\begin{abstract}
The definition of cerebral perfusion pressure (CPP) secondary insults in severe traumatic brain injury remains unclear. The purpose of the present study is to visualize the association of intensity and duration of episodes below or above CPP thresholds and outcome. The analysis was based on prospectively collected minute-by-minute intracranial pressure (ICP) and blood pressure data and outcome from 259 adult patients. The relationship of episodes of CPP below or above a certain threshold for certain duration with the 6-month Glasgow Outcome Score was visualized separately for episodes of active or deficient autoregulation (AR). In adults $\leq 65$ years, an almost exponential transition curve separates the episodes of CPP associated with better outcomes from the episodes of low CPP associated with worse outcomes, indicating that lower CPP could only be tolerated for a brief time. Analysis of episodes of high CPP again showed a time-intensity dependent association with outcome. When combining the two plots, a safe CPP zone between 60 and $70 \mathrm{~mm} \mathrm{Hg}$ could be delineated-however, only for AR active insults. The AR status predominantly affected the transition curve for insults of low CPP. Episodes with ICP $>25 \mathrm{~mm} \mathrm{Hg}$ were associated with poor outcome regardless of CPP. In the present study, the CPP pressure-time burden associated with poor outcome was visualized. A safe zone between 60 and $70 \mathrm{~mm} \mathrm{Hg}$ could be identified for adults $\leq 65$ years, provided AR was active and ICP was $\leq 25 \mathrm{~mm} \mathrm{Hg}$. Deficient AR reduces the tolerability for low CPP.
\end{abstract}

Keywords: adults; cerebral perfusion pressure; cerebrovascular autoregulation; traumatic brain injury

\section{Introduction}

$\mathbf{T}$ RAUMATIC BRAIN INJURY (TBI) is a significant health problem worldwide. In Europe, the overall incidence of fatal and hospitalized TBI is $262 / 100,000 /$ year. ${ }^{1}$ An estimated $43.3 \%$ of hospitalized patients with TBI in the United States experience long-term disability. ${ }^{2}$ The management of severe TBI focuses on avoiding additional ischemic damage from preventable or reversible secondary insults. Intracranial hypertension has long been identified as an insult associated with poorer outcomes. ${ }^{3-5}$ Therefore, it is recommended that intracranial pressure (ICP) be monitored in salvageable patients with severe TBI and to treat patients with ICP above $22 \mathrm{~mm} \mathrm{Hg}^{6}{ }^{6}$ It is evident that " 22 " is not a magical number, and it was recently demonstrated by our group that the relation between ICP level and outcome is not only time dependent, but that ICP tolerability also depends on age and pressure autoregulation (AR) capacity. ${ }^{7}$

While the relation between ICP and outcome is still rather straightforward, however, guidelines on blood pressure management in severe TBI seem more difficult to produce. Cerebral perfusion pressure (CPP) is the driving force of cerebral blood flow, expressed as the difference between the mean arterial blood pressure (MAP) and ICP. Within a certain range of CPP, the cerebral

\footnotetext{
${ }^{1}$ Department of Intensive Care Medicine, University Hospitals Leuven, Leuven, Belgium.

${ }^{2}$ Department of Clinical Physics, Southern General Hospital, Glasgow, United Kingdom.

${ }^{3}$ Department of Perioperative Medicine and Intensive Care Medicine, San Gerardo Hospital, Monza, Italy.

${ }_{5}^{4}$ Medical Physics, James Cook University Hospital, Middlesborough, United Kingdom.

${ }^{5}$ Department of Neurosciences, Neurosurgery, Uppsala, Sweden.

Departments of ${ }^{6}$ Neurosurgery and ${ }^{7}$ Intensive Care Medicine, Antwerp University Hospital, Edegem, Belgium.

${ }^{8}$ Klinik für Neurochirurgie, Universitätsklinikum Tübingen, Tübingen, Germany.

${ }^{9}$ School of Mathematics and Statistics, University of Glasgow, Glasgow, United Kingdom.

${ }^{10}$ Department of Clinical Physics and Bioengineering, NHS Greater Glasgow \& Clyde, Glasgow, United Kingdom.

${ }^{11}$ Department of Neurosurgery, University Hospitals Leuven, Leuven, Belgium.

Fabian Güiza and Geert Meyfroidt contributed equally to this article.
}

(c) Fabian Güiza et al., 2017; Published by Mary Ann Liebert, Inc. This Open Access article is distributed under the terms of the Creative Commons License (http://creativecommons.org/licenses/by/4.0), which permits unrestricted use, distribution, and reproduction in any medium, provided the original work is properly credited. 
vasculature has the ability to maintain constant cerebral blood flow, which is-among others-based on alterations in basal tone and subsequent diameter changes of pial arterioles. ${ }^{8}$ This capacity for "pressure AR" can be deficient after TBI. ${ }^{9}$ Moreover, with intact AR, low CPP can induce a vasodilatory cascade resulting in raised ICP.

The latter phenomenon led Rosner and associates ${ }^{10}$ to formulate the recommendation that CPP in severe TBI situations should be kept above $70 \mathrm{~mm} \mathrm{Hg}$. This concept, however, could not be demonstrated to produce better outcomes in a randomized controlled trial. ${ }^{11}$ At the same time the Lund concept, which emphasized avoidance of the use of vasopressors and accepted CPP down to $50 \mathrm{~mm} \mathrm{Hg}$ for adults, did not result in poorer outcomes. ${ }^{12}$ An explanation for this apparent paradox was sought mainly in the dynamic, and hence more complex, behavior of pressure AR capacity or deficiency after TBI. ${ }^{13,14}$

Real-time monitoring of cerebrovascular pressure reactivity through parameters such as the Pressure Reactivity Index (PRx) ${ }^{14}$ or Low frequency Autoregulation Index $(\mathrm{LAx})^{15}$ has enabled the identification of CPP ranges in which AR is more active, ${ }^{15-17}$ and in retrospective series, higher percentages of time of actual CPP contained within these zones were associated with better outcomes. ${ }^{15,18}$ At present, pressure reactivity monitoring is predominantly performed in centers with a research interest in severe TBI.

In the latest version of the Brain Trauma Foundation guidelines, the chapter on CPP thresholds is limited to a level IIB recommendation that the CPP value to target lies within the range of 60 to $70 \mathrm{~mm} \mathrm{Hg}$. ${ }^{6}$ For a parameter subject to variability such as CPP, however, it may be helpful to the clinician to have more insight into the burden on outcome of CPP insults in terms of severity and duration.

The aim of the present study is to assess the effect of CPP insults, according to varying definitions of intensity and duration, on functional outcome at six months based on prospective data from continuously monitored adult patients with severe TBI. In addition, the impact of ICP, cerebrovascular AR, age, and decompressive craniectomy (DC) on the capacity to tolerate the CPP insults is investigated.

\section{Methods}

\section{Patients and data}

The study population consisted of 259 patients with severe TBI aged 16 years and older:

- There were 164 patients included from the Brain-IT database, ${ }^{19} \mathrm{a}$ multicenter data collection between March 2003 and July 2005 (Newcastle, Uppsala, Monza, Leuven, Heidelberg, Iasi, Barcelona, Leipzig, Kaunas, Turin, Novara, Vilnius, Milan, Glasgow, Mannheim, Edinburgh, Southampton, Cambridge, Zurich, London, Göteborg). The Multi-Centre Research Ethics Committee for Scotland MREC/02/0/9 granted the use of these data for scientific purposes on February 14, 2002.

- The data of the remaining 95 adult patients were collected from four centers: 38 from the San Gerardo Hospital in Monza, Italy, between March 2010 and April 2013; 25 from the University Hospitals, Leuven, Belgium, between September 2010 and September 2013; 20 from the University Hospital, Antwerp, Belgium, between May 2010 and June 2013; and 12 from the University Hospital, Tübingen, Germany, between February and December 2009. Local Ethical Committee approval to use the anonymized data for this analysis was obtained in all centers.
Data collection included baseline risk factors (age, sex, admission Glasgow Coma Score (GCS), admission pupil reactivity), and minute-by-minute ICP and MAP monitoring data. Monitoring data were recorded in minute-by-minute resolution through specifically designed "BrainIT data collection software" for the BrainIT study. ${ }^{19}$ For the later cohort, data were recorded through the centers' Patient Data Management System, in minute-by-minute resolution in Leuven, Tübingen, and Monza, and in second-by-second resolution in Antwerp. For the Antwerp patients, the median value per minute was used in this analysis. Minute-by-minute signals from all data sets were reviewed independently by two senior clinicians in Leuven (GM, BD), and obvious artifacts at visual inspection of data traces were removed.

Finally, a correction of the CPP values for arterial blood pressure transducer height was made based on the information that was obtained on the height of the arterial blood pressure transducer and of the head of the bed. For patients in whom the transducer was at cardiac atrium level and who received nursing care with the head of the bed elevated at 30 degrees, $10 \mathrm{~mm} \mathrm{Hg}$ was subtracted from the registered CPP. This was the case in 101/259 patients (39.0\%).

To investigate the effect of DC and old age on the capacity to tolerate CPP insults, the analyses were performed separately for three groups: patients $\leq 65$ years who did not undergo DC $(n=179)$, patients $>65$ years who did not undergo DC $(n=35)$, and patients $\leq 65$ years who underwent DC $(n=37)$. The details of the three groups are presented in Table 1 . The group of patients $\leq 65$ years who did not undergo DC was considered the standard group on which the analyses assessing the effect of AR and ICP were performed (see below).

\section{Outcome}

Functional outcome was assessed at 6 months in all centers using the Glasgow Outcome Score (GOS). ${ }^{20}$

\section{Visualization method}

The method developed in our group for visualizing the univariate association between insult and outcome and that was used for assessing the pressure and time burden of intracranial hypertension in Güiza and colleagues ${ }^{7}$ was applied in the current analysis investigating the relationship between CPP and outcome. For CPP, two types of insults are defined: insults characterized by CPP dropping below a certain pressure value for a certain duration of time as well as insults characterized by CPP exceeding a certain pressure value for a certain duration of time. Hence, the visualization was performed separately for insults of low CPP and insults of high CPP. The Pearson correlation coefficient between the number of insults of a certain intensity and duration and GOS was expressed by a graded color code: negative correlations in red $(-1$ in dark red) and positive correlations in blue (+1 in dark blue). The contour for zero correlation was highlighted in black and defined as the "transition curve." For more details about the visualization method, we refer to Güiza and coworkers. ${ }^{7}$ Analytic routines were programmed in Matlab ${ }^{\circledR}$ (MathWorks Inc, Natick, MA).

\section{Role of cerebrovascular pressure reactivity expressed by $L A x$}

The LAx, expressing cerebrovascular pressure reactivity as an indicator of pressure AR, is calculated as the moving median of the correlation coefficients between ICP and MAP for the past time intervals of $3,5,10,20,30,60$, and $90 \mathrm{~min}$, using minute-by-minute resolution data. ${ }^{15}$ The LAx was calculated at every minute during the monitoring period and average LAx for every CPP insult period, which allowed for discriminating CPP insults associated with "active" AR (if average LAx $<0$ ) or "passive" AR (if average LAx $\geq 0$ ). Hence, the relationship between CPP insults and outcome was visualized separately considering active or passive episodes exclusively. 
Table 1. Patient Demographic, Injury, and Outcome Data

\begin{tabular}{|c|c|c|c|}
\hline & $\begin{array}{l}\text { Cohort } \leq 65 \text { years } \\
\text { of age without } D C\end{array}$ & $\begin{array}{l}\text { Cohort }>65 \text { years } \\
\text { of age without } D C\end{array}$ & $\begin{array}{c}\text { Cohort } \leq 65 \text { years } \\
\text { of age with DC }\end{array}$ \\
\hline Number of patients $(n)$ & 179 & 35 & 37 \\
\hline LOS days, median (IQR) & $14(7-23)$ & $14(6.5-24.5)$ & $24.5(14-31)$ \\
\hline Age, median (IQR) & $36(24-50.1)$ & $72(69-75.5)$ & $36.9(23-52.3)$ \\
\hline Sex (\% male $)$ & 82.1 & 71.4 & 83.8 \\
\hline \multicolumn{4}{|l|}{ Pupil reactivity } \\
\hline None $(\%)$ & 9.5 & 20.0 & 18.9 \\
\hline One $(\%)$ & 12.3 & 5.7 & 10.8 \\
\hline Two $(\%)$ & 72.1 & 68.6 & 67.6 \\
\hline Unknown, untestable or missing (\%) & 6.1 & 5.7 & 2.7 \\
\hline GCS total, median (IQR) & $7(4-10)$ & $7.5(4.5-11)$ & $7(3-11)$ \\
\hline Unknown, untestable or missing (\%) & 5.6 & 8.6 & 10.8 \\
\hline GCS motor, median (IQR) & $4(1-5)$ & $5(2-5)$ & $4(1-5)$ \\
\hline Unknown, untestable or missing (\%) & 2.8 & 2.7 & 10.8 \\
\hline CPP monitored time (days) & $5.8(2.9-10.7)$ & $5.1(2.7-9)$ & $10.7(5.4-12.2)$ \\
\hline Percent valid CPP monitored time* & $94.9(89.6-97.6)$ & $95.0(83.1-97.6)$ & $93.0(81.1-96.7)$ \\
\hline GOS at six months, median (IQR) & $4(3-5)$ & $3(1-4.8)$ & $4(3-5)$ \\
\hline GOS $1=$ death $(n ; \%)$ & $23 ; 12.9$ & $13 ; 37.1$ & $5 ; 13.5$ \\
\hline GOS $2=$ vegetative $(n ; \%)$ & $4 ; 2.2$ & $3 ; 8.6$ & $2 ; 5.4$ \\
\hline GOS $3=$ severe disability $(n ; \%)$ & $49 ; 27.4$ & $9 ; 25.7$ & $11 ; 29.7$ \\
\hline GOS $4=$ moderate disability $(n ; \%)$ & $43 ; 24.0$ & $1 ; 2.9$ & $4 ; 10.8$ \\
\hline GOS $5=$ low disability $(n ; \%)$ & $60 ; 33.5$ & $9 ; 25.7$ & $15 ; 40.5$ \\
\hline
\end{tabular}

*Non-valid data were because of monitor disconnections, data loss during patient transport. or artefacts removed manually.

DC, decompressive craniectomy; LOS, length of stay; IQR, interquartile range; GCS, Glasgow Coma Scale; CPP, cerebral perfusion pressure; GOS, Glasgow Outcome Scale.

\section{Role of ICP}

The relationship between CPP insults and outcome was visualized separately for CPP insults associated with average ICP $\leq 25 \mathrm{~mm} \mathrm{Hg}$ and for CPP insults associated with average ICP $>25 \mathrm{~mm} \mathrm{Hg}$ to examine the relative effect of ICP and CPP insults on outcome. The value of $25 \mathrm{~mm} \mathrm{Hg}$ was chosen as the discriminator because ICP above $25 \mathrm{~mm} \mathrm{Hg}$ was associated with poor outcome independent of the insult duration in our ICP article. ${ }^{7}$

\section{Multivariate analysis}

Multivariate logistic regression models for death were calculated to investigate the independent association of the cumulative dose of CPP insult burden with outcome. Therefore, the percentage of total monitoring time each patient had CPP insults associated with worse GOS (i.e., time spent in the red zone) was used as an independent variable, together with the IMPACT model core variables age, pupils, and admission Glasgow motor score. ${ }^{21}$ The percentage of monitoring time spent in the red zone was based on a new calculation, in which for each patient, for every minute of monitoring it was checked whether this minute fulfilled criteria of belonging to one or more of the several episodes negatively associated with outcome (i.e., red zone). The time in the red zone was then divided by the total monitoring time of that patient.

\section{Impact of CPP policy}

The database contains patients treated before and after the publication of the third revision of the Brain Trauma Foundation guidelines in 2007, in which the recommendation to aim at CPP higher than $70 \mathrm{~mm} \mathrm{Hg}$ was abandoned. ${ }^{22}$ To assess whether CPP policy confounded the current analysis, the median of the patients' median CPP was calculated per center. For the two centers that contributed data to the cohort before and after 2007, the median was calculated separately for the patients of both periods. Next, the visualization method was applied separately for centers with a median CPP below and above $70 \mathrm{~mm} \mathrm{Hg}$ to assess its validity independent of CPP policy.

\section{Results}

For adults $\leq 65$ years who did not undergo DC ( $n=179)$, the visualization of the association between GOS and the intensity and duration of CPP insults is shown in Figure 1. In both instances (Fig. 1a and Fig. 1b), a blue zone (representing episodes of CPP associated with higher GOS) and red zone (representing episodes of CPP associated with lower GOS) can be observed, separated by a sharply demarcated transition curve. Figure 1a shows that insults below a certain threshold were associated with worse outcomes and that the lower the CPP value, the shorter the duration they could be tolerated.

In the present analysis, for instance, transition to worse outcomes below CPP of $60 \mathrm{~mm} \mathrm{Hg}$ occurred at a duration of $10 \mathrm{~min}$, while $\mathrm{CPP}$ of $50 \mathrm{~mm} \mathrm{Hg}$ could not be tolerated at all. Figure $1 \mathrm{~b}$ shows a similar parabolic course of the association for insults above a certain CPP threshold with transition to worse outcomes occurring at shorter durations for the higher CPP values. When combining both plots (Fig. 1c), it becomes apparent that no universal CPP value is associated with good outcome for longer than $210 \mathrm{~min}$. In other words, a common and static safe CPP zone could not be found. The graphs are cut off at $360 \mathrm{~min}$; they became blurred at longer durations because of the numbers of insults of such long durations steeply falling down.

Figure 2 shows the transition curves for both insults of low CPP and insults of high CPP in adults $\leq 65$ years without DC, separately for insults during which AR was active and insults during which AR was passive (the corresponding color-coded plots are available in Supplementary Fig. 1; see online supplementary material at ftp.liebertpub.com). In active episodes, there was a pronounced shift to the left of the transition curve for low CPP, while the 

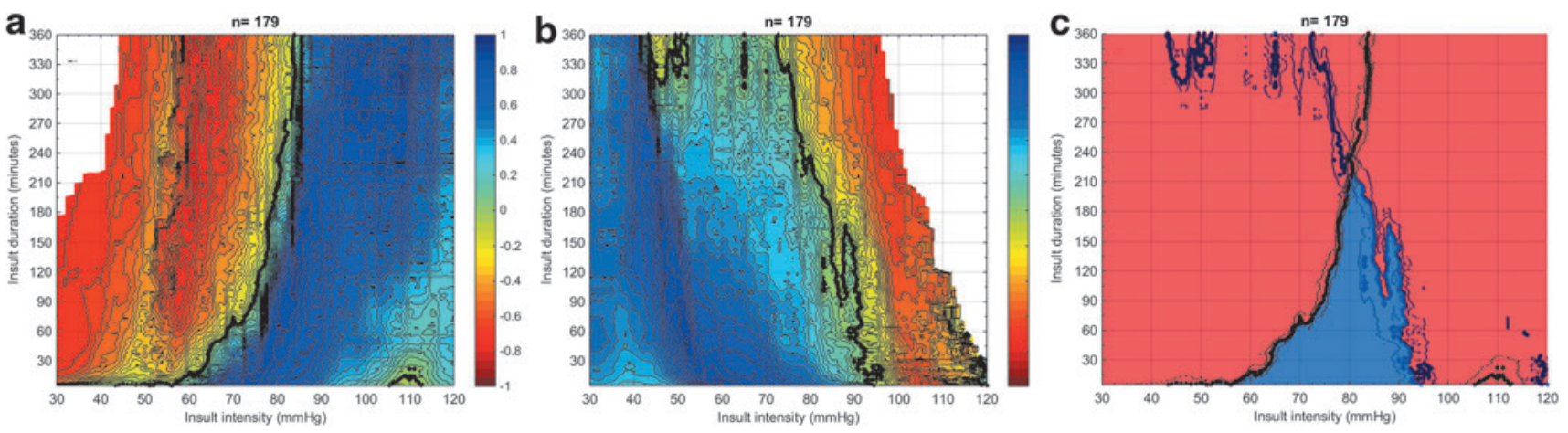

FIG. 1. Visualization of correlation between the Glasgow Outcome Score (GOS) and average number of cerebral perfusion pressure (CPP) insults per GOS category for adults $\leq 65$ years without decompressive craniectomy, $n=179$. Each dot in the graph represents a CPP insult-i.e., an episode of CPP defined by an intensity threshold (X-axis) and a certain duration (Y-axis). The univariate correlation between the average number of a certain CPP insult defined by severity (X-axis) and duration (Y-axis) and each GOS category is colorcoded with blue representing a positive correlation and red representing a negative correlation. The contour of zero correlation is highlighted in black. 1a: insults of low CPP (i.e., CPP on the X-axis expresses the threshold defining insults where CPP went below this threshold for a certain duration). $1 \mathrm{~b}$ : insults of high CPP (i.e., CPP on the X-axis expresses the threshold defining insults where CPP went above this threshold for a certain duration). 1c: low CPP (black) and high CPP (blue) transition curves plotted together.

transition curve for high CPP slightly shifted to the right, resulting in a zone between 60 and $70 \mathrm{~mm} \mathrm{Hg}$ in which CPP was associated with better outcome up to $360 \mathrm{~min}$. In passive episodes, the transition curve for high CPP remained fairly constant, but the transition curve for low CPP showed a pronounced shift to the right, resulting in the finding that no CPP value was associated with good outcome for longer than $90 \mathrm{~min}$.

In Figure 3, the visualization of the relation between GOS and CPP insults for adults $\leq 65$ years without DC is repeated, however, while only selecting episodes where ICP was $\leq 25 \mathrm{~mm} \mathrm{Hg}$ (Fig. 3a and 3c) or only selecting episodes where ICP was $>25 \mathrm{~mm} \mathrm{Hg}$ (Fig. $3 \mathrm{~b}$ and $3 \mathrm{~d}$ ). The graphs for ICP $\leq 25 \mathrm{~mm} \mathrm{Hg}$ are almost identical to the graphs depicting all insults, while the graphs for ICP $>25 \mathrm{~mm} \mathrm{Hg}$ are overwhelmingly red: i.e., all insults are associated with poor outcomes.

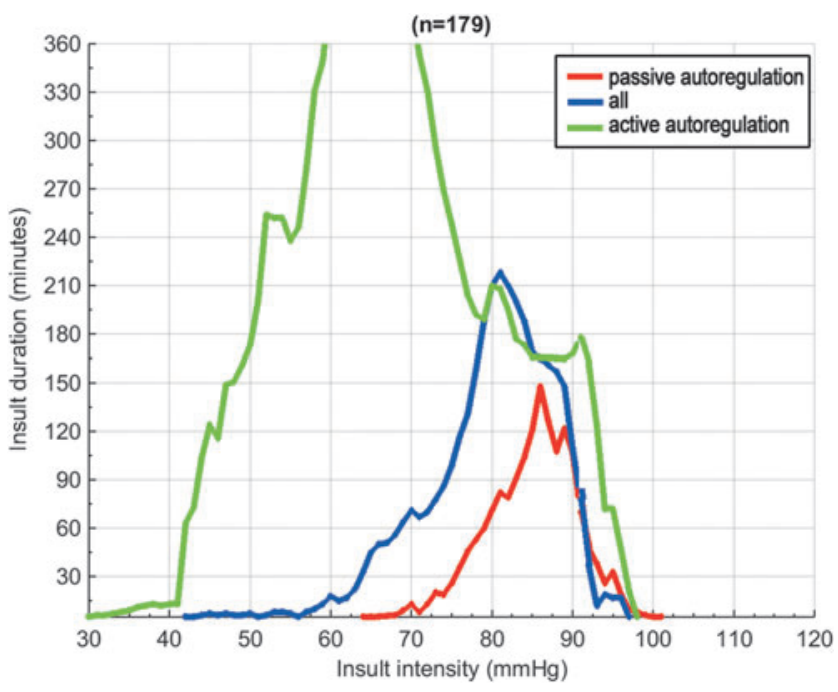

FIG. 2. Comparison of cerebral perfusion pressure insult transition curves for episodes with active autoregulation (LAx $<0$; green) for episodes with passive autoregulation ( $L A x \geq 0$; red) and for all episodes (blue). Transition curves are the lines with 0 correlation between CPP insults and outcome. Analysis performed in adults $\leq 65$ years without decompressive craniectomy, $n=179$.
As for elderly persons $>65$ years without DC $(n=35)$, no transition curves could be discerned, outcomes being overall poor (see Supplementary Fig. 2; see online supplementary material at ftp.liebertpub.com). In adults $\leq 65$ years who underwent a DC $(n=37)$, no meaningful transition curves could be identified either (see Supplementary Fig. 3; see online supplementary material at ftp.liebertpub.com). The percentage of monitoring time with active AR was $44.5 \%$ for the adults $\leq 65$ years without DC and $38.2 \%$ for the elderly without DC.

Nine centers had a median of patients' median CPP below $70 \mathrm{~mm} \mathrm{Hg}$ and 11 above $70 \mathrm{~mm} \mathrm{Hg}$ (Fig. 4). When the color-coded plots visualizing the burden of CPP insults for adults $\leq 65$ years without DC are depicted separately for both groups of centers, the transition curve patterns remain similar (see Supplementary Fig. 4; see online supplementary material at ftp.liebertpub.com).

In the multivariate analysis, the percentage of monitoring time each adult spent in the red zone (based on the transition curves of the active adults $\leq 65$ years without DC) was an independent predictor of mortality (odds ratio [OR] 4.79 [1.02-22.46]; $p=0.04$, Supplementary Table 1; see online supplementary material at ftp.liebertpub.com).

\section{Discussion}

We used the method for visualizing the burden of intracranial hypertension in patients with severe TBI developed by our group ${ }^{7}$ to visualize the burden of CPP insults in terms of poor outcomes associated with episodes in which certain CPP thresholds are exceeded for certain durations. In the group of adults $\leq 65$ years who did not undergo DC, we found that positive and negative associations between the occurrence of insults and outcome were separated by a well demarcated transition curve, which had an exponential course similar to that seen in the ICP study. ${ }^{7}$ The lower the CPP, the shorter the time this could be tolerated, and the same was true for high CPP. Hence, both transition curves for CPP have to be considered together, which results in a safe zone in between the two curves. Only in active AR, however, the curves spread apart sufficiently to result in a CPP zone that was associated with good outcomes for a longer period. In deficient $A R$, the curves came closer, and no CPP value was safe for more than $90 \mathrm{~min}$. 

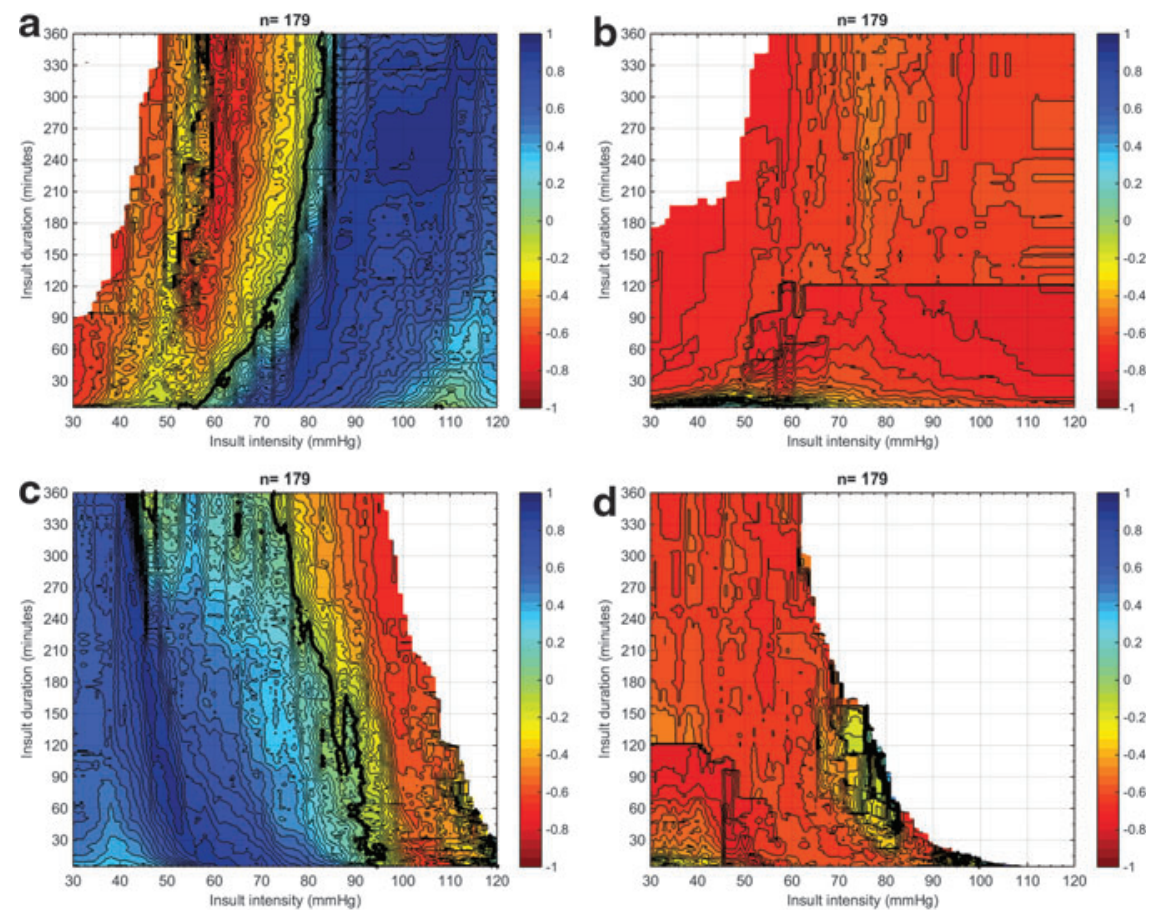

FIG. 3. Visualization of correlation between Glasgow Outcome Scale (GOS) and average number of cerebral perfusion pressure (CPP) insults according to an intracranial pressure (ICP) threshold of $25 \mathrm{~mm} \mathrm{Hg}$. Analysis performed in adults $\leq 65$ years without decompressive craniectomy, $n=179$. The univariate correlation between the average number of a certain CPP insult defined by severity (X-axis) and duration (Y-axis) and each GOS category is color-coded with blue representing a positive correlation and red representing a negative correlation. The contour of zero correlation is highlighted in black. 3a: insults of low CPP for ICP $\leq 25 \mathrm{~mm} \mathrm{Hg}$; $3 \mathrm{~b}$ : insults of low CPP for ICP $>25 \mathrm{~mm} \mathrm{Hg}$; 3c: insults of high CPP for ICP $\leq 25 \mathrm{~mm} \mathrm{Hg}$; 3d: insults of high CPP for ICP $>25 \mathrm{~mm} \mathrm{Hg}$.

Although this finding may have been influenced by unknown confounders, the observation is congruent with the phenomenon of $\mathrm{AR}$ as a stable cerebral blood flow $(\mathrm{CBF})$ plateau regardless of MAP in the Lassen representation. ${ }^{8}$ Strikingly, AR status predominantly affected the transition curve for insults of low CPP. In the older age group and DC group, the sample size was probably too low to draw conclusions. Still, however, it was striking that the graph pattern observed in the younger adults without decompression did not emerge here. When analyzing the total patient cohort including elderly and DC patients, relatively similar graphs as in

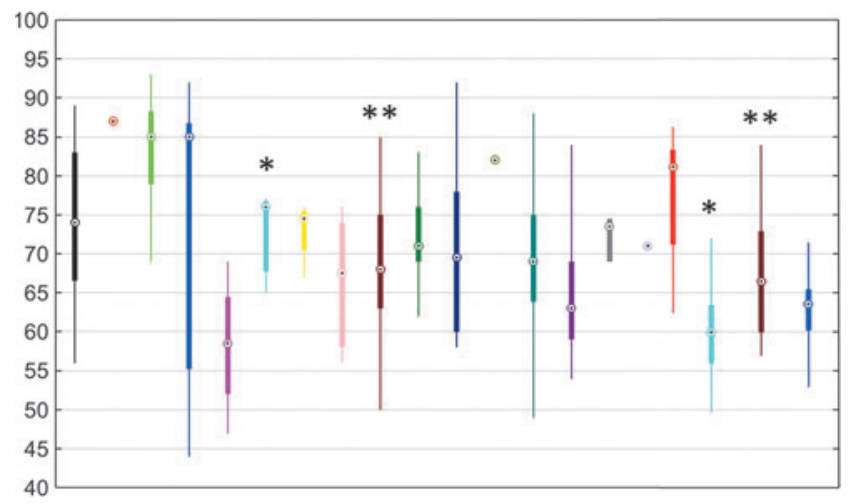

FIG. 4. Per center distribution (median, interquartile range, and total range) of patients' median cerebral perfusion pressure throughout the intensive care unit stay. Each color represents a center. $*$ and $* *$ indicate centers that are depicted twice: once for the cohort 2003-2005 and once for the cohort 2009-2013.
Figure 1 were produced, but with a wider area of transition between good and poor outcome instead of sharper transition curves.

The Lassen representation of the cerebrovascular AR as a CBF plateau for MAP between 60 and $150 \mathrm{~mm} \mathrm{Hg}^{8}$ has been criticized as an oversimplification and more importantly because it was based on a disputable methodology of grouping populations from different studies. ${ }^{23,24}$ From physiological research, AR emerges as a complex feedback mechanism with overlapping and interacting pathways, including intrinsic vasomotor mechanisms, peripheral autonomic vasomotor regulations, and central intrinsic neural pathways. The actual intra-individual range of effective AR is thought to be as narrow as approximately $10 \mathrm{~mm} \mathrm{Hg} .{ }^{25}$ Also, AR would seem to be better at buffering increases versus decreases of MAP, ${ }^{26,27}$ which is inverse to our observation that AR had the most pronounced effect on the "tolerance curve" for low CPP. Noteworthy, the effect of AR on the transition curve for low CPP in the present analysis concerns a wider pressure range than the effect of AR on the ICP transition curve in Güiza and associates. $^{7}$

In several studies, it has been reported that the impairment of AR in TBI is a dynamic phenomenon. ${ }^{14,28,29}$ In active AR, a safe CPP zone between 60 and $70 \mathrm{~mm} \mathrm{Hg}$ for up to $6 \mathrm{~h}$ is depicted in the current study. How this safe zone looks like for longer periods cannot be concluded from the present study. Variability of blood pressure and ICP in real life, as in the present study, however, may question the relevance of this issue. The safe zone is congruent with the AR stable CBF plateau concept, but a dynamic component is added-i.e., the actual range of safe CPP is wider than 60 to $70 \mathrm{~mm}$ $\mathrm{Hg}$, but outside the 60 to $70 \mathrm{~mm} \mathrm{Hg}$, it is time dependent.

This is not in conflict with the literature on optimal CPP. ${ }^{16-18}$ First, there is a different perspective. The optimal CPP method 
searches for CPP values associated with active AR (based on PRx or LAx) and then finds a relationship with outcome. The current study investigated the relationship between $\mathrm{CPP}$ and outcome and then looked at the influence of AR (using LAx). Second, associations with favorable outcome can be found far outside the 60 to $70 \mathrm{~mm} \mathrm{Hg}$ range, but then they are time dependent. The optimal $\mathrm{CPP}$ method, within the scope of the CPP range the patient has been in, will pick up time-dependent CPP zones associated with active AR in this wider range. Future studies, both clinical studies as well as validation studies of clinical tools in animal AR models, will be required to refine our understanding of $\mathrm{AR}$ and optimal $\mathrm{CPP}$. What can be concluded from the current article and our ICP article ${ }^{7}$ is that active AR improves the tolerability for insults of high ICP and low CPP. Consequently, it is likely beneficial to bring the patient with TBI in a state of active AR.

Insults of CPP $<50 \mathrm{~mm} \mathrm{Hg}$ were hardly tolerated, and ICP $>25 \mathrm{~mm} \mathrm{Hg}$ was associated with poor outcome regardless of CPP. The latter two findings were also demonstrated in Güiza and coworkers. $^{7}$ That the impact of high ICP on outcome overrules the effect of CPP, however, should not be interpreted as if the management of CPP is unimportant during episodes of high ICP. It may well be that, more than the pressure level itself, high ICP reflects the global seriousness of the patient's condition, and hence is reflected in its profound relationship with poor outcome.

The current study inevitably has limitations. First, the sample size remains relatively small. Second, the data incorporate therapeutic influences, which cannot be removed. As we have shown, however, CPP policies did not affect the CPP/outcome transition curves. Third, although efforts were made to correct for the height at which arterial blood pressure was zeroed, some degree of uncertainty cannot be excluded. When we analyzed the data without correction, however, the influence on the graphs was minimal and the interpretation remained similar. Fourth, longer-staying patients have a proportionally higher representation in the total monitoring time. To rule out bias from this phenomenon, the visualization plots were recalculated using daily averages of the number of insults. The graphs remained unchanged (Supplementary Fig. 5; see online supplementary material at ftp .liebertpub.com). Fifth, artifacts in the monitoring data were manually removed by two clinical experts, and we cannot exclude that some artifacts went unnoticed. Sixth, although the results seemed robust, we cannot exclude an influence from confounders that were not analyzed. Also, it should be kept in mind that this analysis reports on correlative and not necessarily causative relationships. Seventh, AR was considered as active (LAx negative) or passive (LAx positive), while in reality it does not behave as an on-off phenomenon, and the threshold of " 0 " is debatable. Moreover, it should be taken into account that LAx produces a rough estimation of changes of AR capacity over longer periods, with lower resolution than the actual phenomenon. Finally, LAx may behave noisy when only considered in short time intervals, which should be taken into account when interpreting the influence of AR on the very short CPP insult periods.

The present study attempts to produce a better insight into insults of low and high CPP. The results support that there is an impact of AR on the relationship between CPP and outcome and demonstrate the important influence of the time factor. From this, it is impossible to produce fixed CPP thresholds. This emphasizes the need for improving our understanding of cerebrovascular pathophysiology in TBI and including this knowledge into smart(er) monitoring tools.

\section{Acknowledgments}

This study is funded through Fonds voor Wetenschappelijk Onderzoek Vlaanderen (Research Project G.0904.11). Geert Meyfroidt receives funding from Fonds voor Wetenschappelijk Onderzoek Vlaanderen as senior clinical investigator (1846113N), Greet Van den Berghe receives structural research funding via the Methusalem program funded by the Flemish Governement (METH/08/07). Brain-IT was funded by the European Framework Program (FP5QRLI-2000-00454), QLGT-2002-00160 and FP7-IST-2007-217049). The NEMO project in the Universty Hospital Antwerp was funded by the Flemish Governement Agency for Innovation by Science and Technology (IWT). The authors wish to acknowledge the non-author steering group members of Brain-IT: Barbara Gregson, Tim Howells, Karl Kiening, Arminas Ragauskas, Juan Sahuquillo, and Jan Oliver Neumann.

\section{Author Disclosure Statement}

No competing financial interests exist.

\section{References}

1. Peeters, W., van den Brande, R., Polinder, S., Brazinova, A., Steyerberg, E.W., Lingsma, H.F., and Maas, A.I. (2015). Epidemiology of traumatic brain injury in Europe. Acta Neurochir. (Wien) 157, 1683-1696.

2. Selassie, A.W., Zaloshnja, E., Langlois, J.A., Miller, T., Jones, P., and Steiner, C. (2008). Incidence of long-term disability following traumatic brain injury hospitalization, United States. J. Head Trauma Rehabil. 23, 123-131.

3. Narayan, R.K., Kishore, P.R., Becker, D.P., Ward, J.D., Enas, G.G., Greenberg, R.P., Domingues Da Silva, A., Lipper, M.H., Choi, S.C., Mayhall, C.G., Lutz, H.A. 3rd, and Young, H.F. (1982). Intracranial pressure: to monitor or not to monitor? A review of our experience with severe head injury. J. Neurosurg. 56, 650-659.

4. Marmarou, A., Anderson, R.L., Ward, J.D., Choi, S.C., Young, H.F., Eisenberg, H.M., Foulkes, M.A., Marshall, L.F., and Jane, J.A. (1991). Impact of ICP instability and hypotension on outcome in patients with severe head trauma. J. Neurosurg. 75, S59-S66.

5. Saul, T.G., and Ducker, T.B. (1982). Effect of intracranial pressure monitoring and aggressive treatment on mortality in severe head injury. J. Neurosurg. 56, 498-503.

6. Carney, N., Totten, A.M., O'Reilly, C., Ullman, J.S., Hawryluk, G.W., Bell, M.J., Bratton, S.L., Chesnut, R., Harris, O.A., Kissoon, N., Rubiano, A.M., Shutter, L., Tasker, R.C., Vavilala, M.S., Wilberger, J., Wright, D.W., and Ghajar, J. (2017) Guidelines for the management of severe traumatic brain injury, Fourth Edition. Neurosurgery 80, 6-15.

7. Güiza, F., Depreitere, B., Piper, I., Citerio, G., Chambers, I., Jones, P.A., Lo, T.Y., Enblad, P., Nillson, P., Feyen, B., Jorens, P., Maas, A., Schuhmann, M.U., Donald, R., Moss, L., Van den Berghe, G., and Meyfroidt, G. (2015) Visualizing the pressure and time burden of intracranial hypertension in adult and paediatric traumatic brain injury. Intensive Care Med. 41, 1067-1076.

8. Lassen, N.A. (1959). Cerebral blood flow and oxygen consumption in men. Physiol. Rev. 39, 183-238.

9. Bouma, G.J., Muizelaar, J.P., Bandoh, K., and Marmarou, A. (1992). Blood pressure and intracranial pressure-volume dynamics in severe head injury: relationship with cerebral blood flow. J. Neurosurg. 77, $15-19$.

10. Rosner, M.J., and Daughton, S. (1990). Cerebral perfusion pressure management in head injury. J. Trauma 30, 933-940.

11. Robertson, C., Valadka, A.B., Hannay, H.J., Contant, C.F., Gopinath, S.P., Cormio, M., Uzura, M., and Grossman, R.G. (1999). Prevention of secondary ischemic insults after severe head injury. Crit. Care Med. 27, 2086-2095.

12. Naredi, S., Olivecrona, M., Lindgren, C., Ostlund, A.L., Grände, P.O., and Koskinen, L.O. (2001). An outcome study of severe traumatic head injury using the "Lund therapy" with low-dose prostacyclin. Acta Anaesthesiol. Scand. 45, 402-406.

13. Howells, T., Elf, K., Jones, P.A., Ronne-Engström, E., Piper, I., Nilsson, P., Andrews, P., and Enblad, P. (2005). Pressure reactivity as 
a guide in the treatment of cerebral perfusion pressure in patients with brain trauma. J. Neurosurg. 102, 311-317.

14. Czosnyka, M., Smielewski, P., Kirkpatrick, P., Laing, R.J., Menon, D., and Pickard, J.D. (1997). Continuous assessment of the cerebral vasomotor reactivity in head injury. Neurosurgery 41, 11-17.

15. Depreitere, B., Güiza, F., Van den Berghe, G., Schuhmann, M.U., Maier, G., Piper, I., and Meyfroidt, G. (2014). Pressure autoregulation monitoring and cerebral perfusion pressure target recommendation in patients with severe traumatic brain injury based on minute-by-minute monitoring data. J. Neurosurg. 120, 1451-1457.

16. Steiner, L.A., Czosnyka, M., Piechnik, K., Smielewski, P., Chatfield, D., Menon, D.K., and Pickard, J.D. (2002). Continuous monitoring of cerebrovascular pressure reactivity allows determination of optimal cerebral perfusion pressure in patients with traumatic brain injury. Crit. Care Med. 30, 733-738.

17. Zweifel, C., Lavinio, A., Steiner, L.A., Radolovich, D., Smielewski, P., Timofeev, I., Hiler, M., Balestreri, M., Kirkpatrick, P.J., Pickard, J.D., Hutchinson, P., and Czosnyka, M. (2008). Continuous monitoring of cerebrovascular pressure reactivity in patients with head injury. Neurosurg. Focus 25, E2.

18. Aries, M.J., Czosnyka, M., Budohoski, K.P., Steiner, L.A., Lavinio, A., Kolias, A.G., Hutchinson, P.J., Brady, K.M., Menon, D.K., Pickard, J.D., and Smielewski, P. (2012). Continuous determination of optimal cerebral perfusion pressure in traumatic brain injury. Crit. Care Med. 40, 2456-2463.

19. Piper, I., Citerio, G., Chambers, I., Contant, C., Enblad, P., Fiddes, H., Howells, T., Kiening, K., Nilsson, P., and Yau, Y.H. (2003). The Brain-IT group: concept and core dataset definition. Acta Neurochirurg. (Wien) 145, 615-628.

20. Jennett, B., and Bond, M. (1975). Assessment of outcome after severe brain damage. A practical scale. Lancet 1, 480-484.

21. Steyerberg, E.W., Mushkudiani, N., Perel, P., Butcher, I., Lu, J., McHugh, G.S., Murray, G.D., Marmarou, A., Roberts, I., Habbema, J.D., and Maas, A.I. (2008). Predicting outcome after traumatic brain injury: development and international validation of prognostic scores based on admission characteristics. PLoS Med. 5, e165.

22. Brain Trauma Foundation; American Association of Neurological Surgeons; Congress of Neurological Surgeons; Joint Section on
Neurotrauma and Critical Care, AANS/CNS, Bratton SL, Chestnut RM, Ghajar J, McConnell Hammond FF, Harris OA, Hartl R, Manley GT, Nemecek A, Newell DW, Rosenthal G, Schouten J, Shutter L, Timmons SD, Ullman JS, Videtta W, Wilberger JE, and Wright DW. (2007). Guidelines for the management of severe traumatic brain injury. IX. Cerebral perfusion thresholds. J. Neurotrauma 24, S59-S64.

23. Drummond, J.C. (1997). The lower limit of autoregulation: time to revise our thinking? Anesthesiology 86, 1431-1433.

24. Willie, C.K., Tzeng, Y.C., Fisher, J.A., and Ainslie, P.N. (2014). Integrative regulation of human brain blood flow. J. Physiol. 592, 841-859.

25. Tan, C.O. (2012). Defining the characteristic relationship between arterial pressure and cerebral flow. J. Appl. Physiol. 113, 1194-1200.

26. Numan, T., Bain, A.R., Hoiland, R.L., Smirl, J.D., Lewis, N.C., and Ainslie, P.N. (2014). Static autoregulation in humans: a review and reanalysis. Med. Eng. Phys. 36, 1487-1495.

27. Tzeng, Y.C., Willie, C.K., Atkinson, G., Lucas, S.J., Wong, A., and Ainslie, P.N. (2010). Cerebrovascular regulation during transient hypotension and hypertension in humans. Hypertension 56, 268-273.

28. Bouma, G., Muizelaar, J.P., Stringer, W.A., Choi, S.C., Fatouros, P., and Young, H.F. (1992). Ultra-early evaluation of regional cerebral blood flow in severely head-injured patients using xenon-enhanced computerized tomography. J. Neurosurg. 77, 360-368.

29. Sviri, G.E., Aaslid, R., Douville, C.M., Moore, A., and Newell, D.W. (2009). Time course for autoregulation recovery following severe traumatic brain injury. J. Neurosurg. 111, 695-700.

Address correspondence to:

Bart Depreitere, $M D, P h D$

Department of Neurosurgery

University Hospitals Leuven

Herestraat 49

3000 Leuven

Belgium

E-mail: bart.depreitere@uzleuven.be 